\title{
The Analysis of Serious Fatal Road Traffic Accidents in China
}

\author{
Miaomiao Liu ${ }^{1}$, Yongsheng Chen ${ }^{1}$ and Guangquan $\mathrm{Lu}^{2}$ \\ ${ }^{1}$ Research Institute of Highway, Ministry of Transport, 8 Xitucheng Road, Haidian District, Beijing 100088, China \\ ${ }^{2}$ School of Transportation Science and Engineering, Beihang University, 37 Xueyuan Road, Haidian District, Beijing 100191, China
}

\begin{abstract}
A large number of road traffic accidents occur each year in China, which results in huge losses to the safety of people's lives and property. Among all accidents, the accident with over 10 fatalities in one accident is extraordinarily serious, which has immeasurable impact on the security. In this paper, we defined these accidents as serious fatal road traffic accidents (SF-RTAs). The aim of this study was to analyze SF-RTAs in China and its provinces and investigate the causes of SF-RTAs. Firstly, we described and analyzed the annual trend of SF-RTAs based on the data from 1990 to 2014 . Then we analyzed region, time and accident type distribution characteristics of SF-RTAs during 2004-2014. Then the main factors influencing SF-RTAs were analyzed by linear regression and decision tree methods. Finally, we proposed corresponding control strategies for preventing SF-RTAs according to the analysis results. It is expected that the findings of this study are useful in indentifying effective prevention measures for serious fatal accidents in China, and protecting human lives and properties.
\end{abstract}

\section{Introduction}

Global Status Report on Road Safety 2015, reflecting information from 180 countries, indicates that worldwide the total number of road traffic deaths remains unacceptably high at 1.25 million per year, and non-fatal injuries as a result of road traffic crashes are between 20 to 50 million. These deaths and injuries have an immeasurable impact on people's lives and property safety. Currently, road traffic accidents are estimated to be the 8th position among the 100 important causes of death. However, it is reported that road traffic injuries will become the 5th leading cause of death by the year 2030 [1]. According to previous documents, traffic accidents on roads depend on many factors, such as changes in seasons, weather conditions, road traffic volume, traffic intensity, driving experience, driving culture and technical state [2]. All these factors are related to traffic safety, human lives and health. Hence, it is essential to analyze the various aspects of road traffic accidents through detailed research.

According to the statistics [3], 6.76 million road traffic accidents occur in China in the year 2014. Among them, 196812 accidents involve fatality or injury, which make 58523 persons killed and 211882 injured. From the year 1990 to 2014, more than 8.88 million accidents involved fatality or injury occur in China, which kill more than 1.90 million people and wound more than 7.29 million people. And these accidents cause huge losses to the safety of people's lives and property. Among all accidents, the accident with over 10 fatalities in one accident is extraordinarily serious, which has immeasurable impact on the safety. We define these accidents as serious fatal road traffic accidents (SF-RTAs) in this paper. 1084 SFRTAs occur in China from 1990 to 2014, which result in 17317 deaths and 18106 injuries (as shown in Fig. 1).

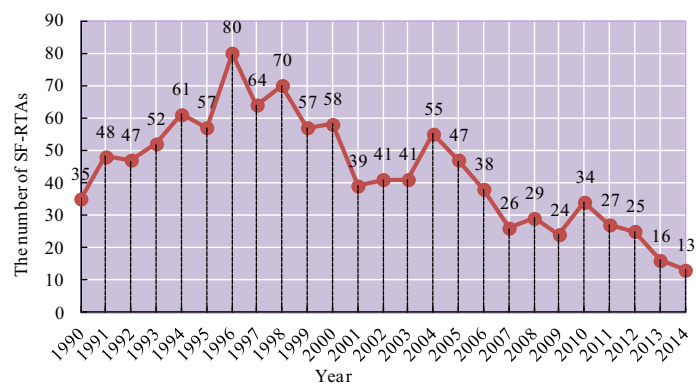

Figure 1. The number of SF-RTAs occurred in China during 1990 and 2014.

These SF-RTAs usually cause a lot of casualties, bring in directly or indirectly property losses, and have serious social impact. Although some traffic safety administration departments of China have taken some measures to prevent SF-RTAs, SF-RTAs still occur from time to time. In addition, the now existing prevention methods lack indepth analysis for the characteristics of SF-RTAs. Thus, in this study, we mainly focused on the characteristics of SF-RTAs occurred in China, investigated the main factors influencing SF-RTAs, and proposed effective measures for preventing SF-RTAs.

\section{Materials}

All SF-RTAs information can be collected by Road Accident Statistics Annual Report of the People's 
Republic of China, but it lacks detailed description in the Annual Report for each of SF-RTA before the year 2003. Detailed information, including date, time, province, address, the number of deaths and injuries, road grade, accident type, vehicle type and vehicle owner of SF-RTA is recorded in Annual Report from 2004. Thus, in this study, we mainly collected and analyzed the SF-RTAs occurred in China during January 2004 and December 2014 [3]. To investigate the main factors influencing SFRTAs, we also collected much data information involved human, vehicle, road and society. Then we analyzed the correlation between the SF-RTAs and influence factors using the statistical software SPSS and data analysis methods.

\section{Methodology and results}

\subsection{Data statistics}

It was known from Fig. 1 that, since 1990s, the number of SF-RTAs every year decreased after increasing with two obvious peaks, 80 SF-RTAs occurred in 1996 and 55 SFRTAs in 2004. From 2004 to 2007, the number of SFRTAs decreased greatly with the improvement of overall road safety in China. Then the number of SF-RTAs increased slightly in 2008 and 29 SF-RTAs happened, killing 476 and injuring 504(as shown in Table 1). In 2009, the number of SF-RTAs decreased to 24. But in 2010, that number surged to 34 with 461 deaths and 432 injuries. Since 2010, the number of SF-RTAs starts to decrease again. In 2014, 13 SF-RTAs happened, killing 229 and injuring 137. Number of SF-RTAs and injuries reached to the historical low in 2014.

Table 1. SF-RTAs occurred in China during 2004 and 2013

\begin{tabular}{|c|c|c|c|}
\hline Year & $\begin{array}{c}\text { The number of } \\
\text { SF-RTAs }\end{array}$ & $\begin{array}{c}\text { The number of } \\
\text { deaths }\end{array}$ & $\begin{array}{c}\text { The number of } \\
\text { injuries }\end{array}$ \\
\hline 2004 & 55 & 852 & 877 \\
\hline 2005 & 47 & 807 & 705 \\
\hline 2006 & 38 & 558 & 463 \\
\hline 2007 & 26 & 389 & 449 \\
\hline 2008 & 29 & 476 & 504 \\
\hline 2009 & 24 & 329 & 345 \\
\hline 2010 & 34 & 461 & 432 \\
\hline 2011 & 27 & 455 & 403 \\
\hline 2012 & 25 & 361 & 367 \\
\hline 2013 & 16 & 208 & 259 \\
\hline 2014 & 13 & 229 & 137 \\
\hline Total & 334 & 5125 & 4941 \\
\hline
\end{tabular}

The accidents data in Fig. 1 and Table 1 show that China is standing at a high frequent stage of SF-RTAs. Thus, it is important to analyze the distribution characteristics of SF-RTAs and then make more efforts to reduce the number of SF-RTAs.

\subsubsection{Regional distribution of SF-RTAs}

The statistics data indicated that SF-RTAs occurred in different regions of China. The number of SF-RTAs in different regions and different provinces from 2004 to 2014 is shown in Fig. 2 and Fig. 3, respectively.

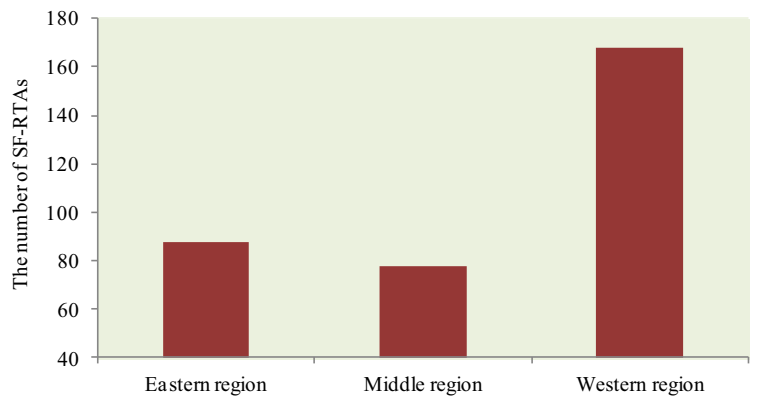

Figure 2. The number of SF-RTAs in different regions from 2004 to 2014

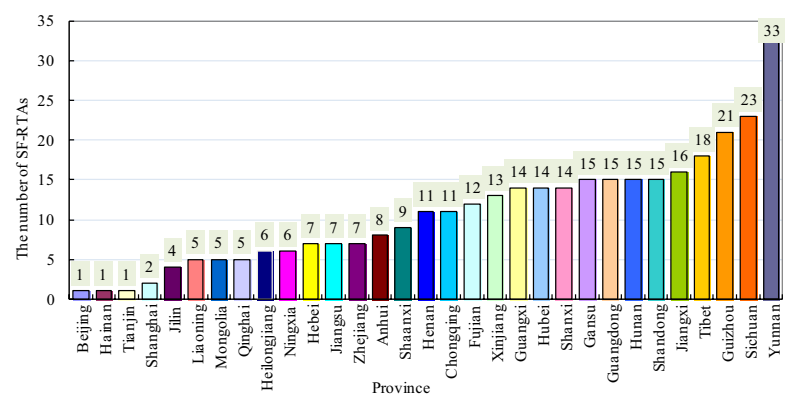

Figure 3. The number of SF-RTAs in different provinces from 2004 to 2014

It was known from Fig. 2 and Fig. 3 that the distribution of SF-RTAs had obvious regional features. Number of SF-RTAs in western region was more than that of eastern and middle regions. From 2004 to 2014, SF-RTAs occurred in Yunnan province was the most, followed by Sichuan and Guizhou provinces. All of these provinces are in south-west region of China. The total number of SF-RTAs happened in these three provinces above was 77 , accounting for $23.1 \%$ of the total number of SF-RTAs. In addition, the number of SF-RTAs occurred in Beijing, Hainan, and Tianjin was the least (was all 1), and these provinces are all in eastern region.

\subsubsection{Time distribution of SF-RTAs}

In order to know the time distribution of SF-RTAs, 334 SF-RTAs occurred during the year 2004 and 2014 were analyzed (Fig. 4-Fig. 6). Fig. 4 shows the hour distribution of SF-RTAs.

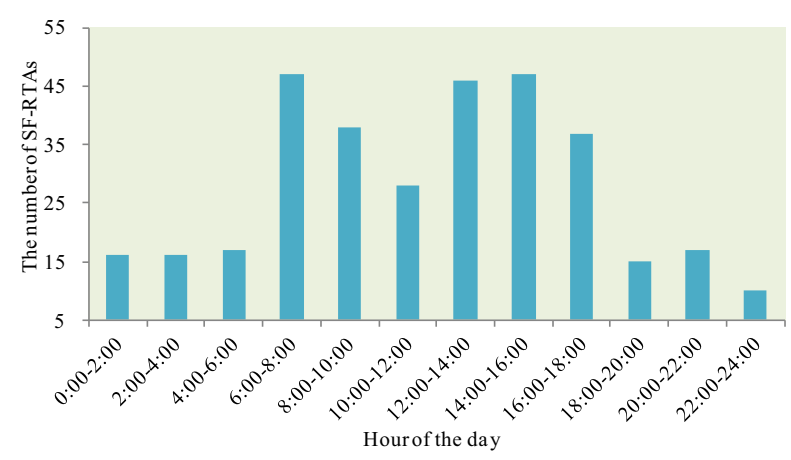

Figure 4. The hour distribution of SF-RTAs

It was known from Fig.4 that the number of SF-RTAs happened between $6: 00$ to $8: 00$ and between 12:00 to 16:00 was more than other time periods. During 22:00 
and 24:00, SF-RTAs were the least. Fig. 5 shows the weekday distribution of SF-RTAs.

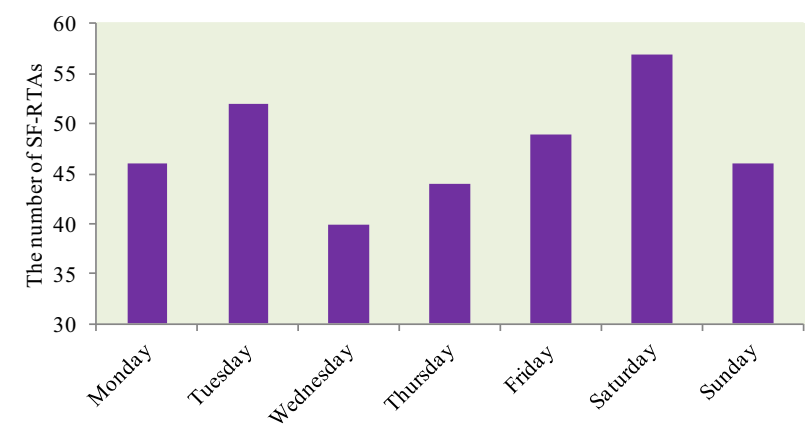

Day of the week

Figure 5. The weekday distribution of SF-RTAs

According to Fig.5, the number of SF-RTAs occurred on Saturday was the most, followed by Tuesday and Friday. On Wednesday, the number of SF-RTAs was the least. Fig. 6 shows the month distribution of SF-RTAs.

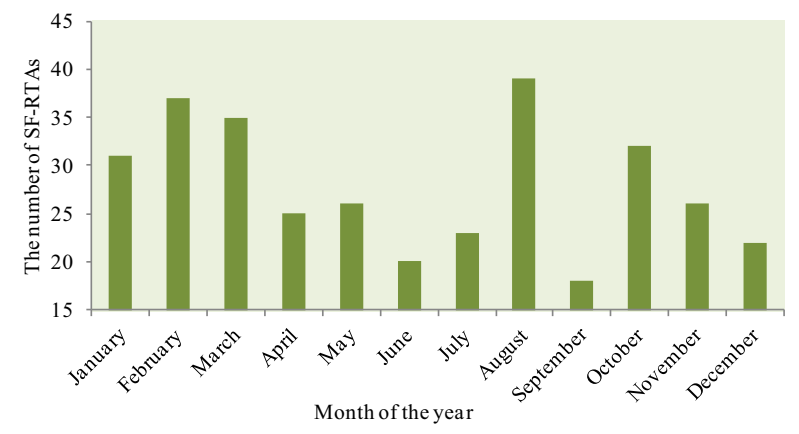

Figure 6. The month distribution of SF-RTAs

It was seen from Fig. 6 that SF-RTAs were more likely to occur in August, followed by February and March. The number of SF-RTAs occurred in September was the least.

\subsubsection{Accident type distribution of SF-RTAs}

SF-RTAs can be divided into many types, including collision, facing scrape, turnover, hit on fixed objects, fire, run-off-road, rolling compaction and explosion, etc. Of which, the collision accidents include head-on collision, offset collision and rear-end collision. And the run-offroad accidents include falling to cliffs, valleys, rivers and bridges. The accident type distribution of SF-RTAs during 2004 and 2014 is shown in Fig. 7.

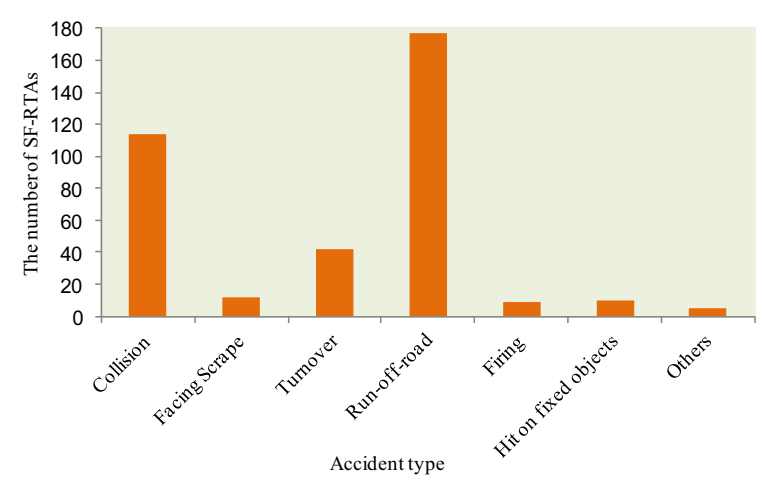

Figure 7. The accident type distribution of SF-RTAs
Fig. 7 indicated the SF-RTAs related to run-off-road were the most, accounting for about $48.0 \%$ of the total number of SF-RTAs. And the number of SF-RTAs related to collision was the second most.

\subsubsection{Other distribution characteristic of SF-RTA}

Vehicle type The distribution of vehicle type related to SF-RTAs is shown in Fig. 8.

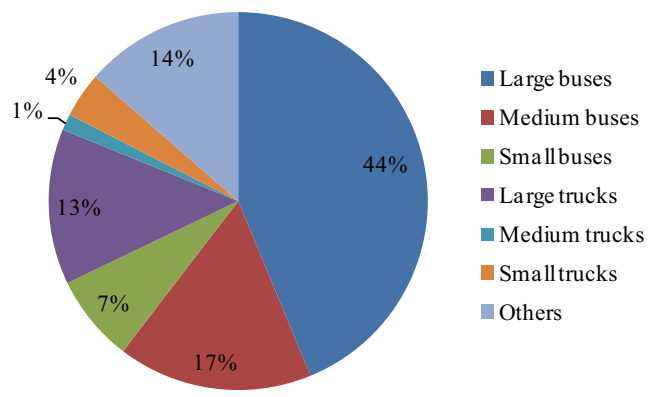

Figure 8. The distribution of vehicle types causing SF-RTAs

Fig. 8 showed that $44 \%$ of the SF-RTAs related to large buses, and $17 \%$ related to medium buses. That is, the SF-RTAs related to large buses and medium buses accounted for more than $60 \%$ of the total. It could be concluded that large buses and medium buses were main vehicle types which easily resulted in SF-RTAs. Road type The distribution of road types related to SF-RTAs is shown in Fig. 9.

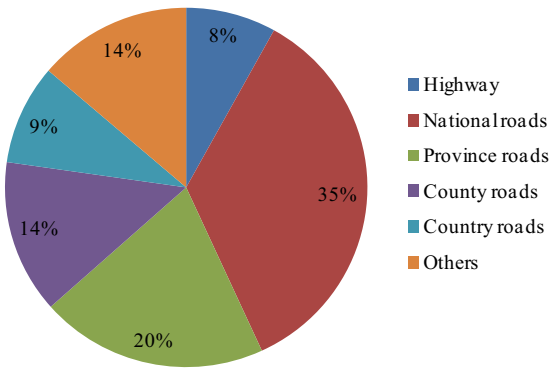

Figure 9. The distribution of road types related to SF-RTAs

It could be seen from Fig. 9 that, for the 334 SF-RTAs from 2004 to 2014 , the accidents related to national roads, province roads and county roads accounted for $35 \%, 20 \%$ and $14 \%$, respectively. That is, SF-RTAs were more likely to happen on national roads and province roads.

Table 2. The factors we chose to analyze SF-RTAs

\begin{tabular}{|l|l|l|}
\hline $\begin{array}{c}\text { Different } \\
\text { types of } \\
\text { factors }\end{array}$ & Variables & \multicolumn{1}{c|}{ Definition } \\
\hline \multirow{3}{*}{ Human } & H1 & The total population \\
\cline { 2 - 3 } & H2 & The number of motor vehicle drivers \\
\cline { 2 - 3 } & H3 & The number of car drivers \\
\hline \multirow{2}{*}{ Vehicle } & V1 & The number of commercial vehicles \\
\cline { 2 - 3 } & V2 & The number of passengers cars \\
\hline & V3 & The number of trucks \\
\hline \multirow{3}{*}{ Road } & R1 & Highway mileage \\
\cline { 2 - 3 } & R2 & Expressway mileage \\
\cline { 2 - 3 } & R3 & Grade highway mileage \\
\cline { 2 - 3 } & R4 & Substandard highway mileage \\
\hline \multirow{2}{*}{ Society } & S1 & Gross domestic product (GDP) \\
\hline
\end{tabular}




\subsection{Main Factors Influencing SF-RTAs}

As we all known, many factors relate to human, vehicle, road and society may have effect on SF-RTAs. Based on the data available, these factors we can obtain are shown in Table 2.

To better understand the main factors influencing SFRTAs, we chose two analysis methods to analyze the relationship between the number of SF-RTAs and different factors in this paper, which included regression analysis and classification tree analysis.

\subsubsection{Regression analysis}

In the traffic field, the application of regression analysis is extensive [4], [5]. In this paper, we chose linear regression module of SPSS to analyze the main factors that affected SF-RTAs in different provinces. The number of SF-RTAs occurred in different province in each year was considered as dependent variable. The factors in Table 2 were independent variables. Then, for each province, the correlation between the number of SF-RTAs and various factors (including human, vehicle, road and society) could be identified. Table 3 shows the variables entered the established model for each province.

Table 3. Variables entered the model for each province

\begin{tabular}{|l|l|l|l|}
\hline \multicolumn{2}{|c|}{ Province } & \multicolumn{2}{c|}{ Province } \\
\hline Name & \multicolumn{1}{|l}{ Variables Entered } & Name & \multicolumn{1}{|c|}{ Variables Entered } \\
\hline \multirow{2}{*}{ Beijing } & $\begin{array}{l}\text { S1, H1, H2, V2, } \\
\text { V3, R1, R2, R4 }\end{array}$ & Shanxi & $\begin{array}{l}\text { S1, H1, H2, H3, V1, } \\
\text { V2, R2, R3, R4 }\end{array}$ \\
\hline \multirow{2}{*}{ Tianjin } & $\begin{array}{l}\text { S1, H1, H2, H3, } \\
\text { V2, V3, R1, R2, R4 }\end{array}$ & Mongolia & $\begin{array}{l}\text { S1, H1, H2, H3, V1, } \\
\text { V2, R1, R2, R4 }\end{array}$ \\
\hline \multirow{2}{*}{ Hebei } & $\begin{array}{l}\text { S1, H1, H2, V1, } \\
\text { V2, R1, R2, R4 }\end{array}$ & Liaoning & $\begin{array}{l}\text { S1, H1, H2, H3, V1, } \\
\text { V2, R2, R3, R4 }\end{array}$ \\
\hline$\ldots \ldots$ & $\ldots \ldots$ & $\ldots \ldots$ & $\ldots \ldots$ \\
\hline
\end{tabular}

The results showed that, S1, H1 and R4 (denoted gross domestic product, the total population and substandard highway mileage, respectively) almost entered all regression models. This indicated that these 3 variables had larger impact on the number of SF-RTAs occurred in China than other factors. In addition, there existed significantly positive relationships between the number of SF-RTAs and H1 as well as R4 for all linear regression models.

\subsubsection{Decision tree analysis}

Decision tree analysis is a popular statistical method which emerges from the field of machine learning and data mining [6]-[8]. In the traffic field, the application of decision trees is also extensive [10], [11]. However, no study has been conducted to date on the analysis of SFRTAs in China. Thus, in this study, we also chose decision tree method to analyze the influence of different factors on SF-RTAs. The main factors influencing the number of SF-RTAs can be obtained by the analysis for root node and leaf nodes in the decision tree results. Similarly, the number of SF-RTAs occurred in different province in each year was the dependent variable of the decision tree model. The factors shown in Table 2 were the independent variables. Fig. 10 shows the result of the established decision tree model.

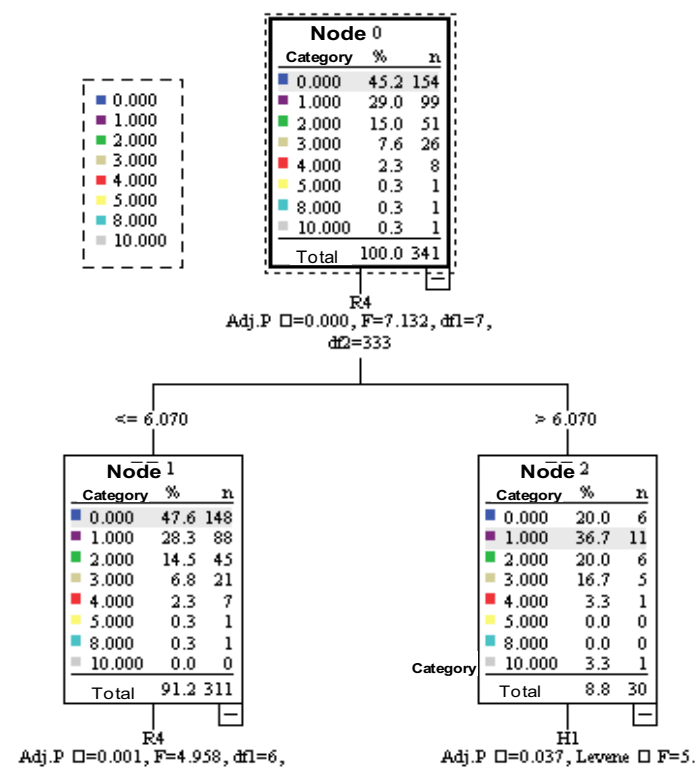

Figure 10. The result of the established decision tree model

According to Fig. 10, the main factors that affected the number of SF-RTAs were R4 (the variable occurred in Node 0 and Node 1) and H1 (the variable occurred in Node 3). This result was similar to the result of regression analysis above. It showed that if we wanted to control the number of SF-RTAs, we should focus on R4 (substandard highway mileage) and H1 (the total population) in each province. Additional detailed descriptions of these decision tree algorithms were beyond the scope of the present study. For further discussions of the tree methodology, please refer to the study of Breiman et al. [9].

\section{Discussion and conclusion}

According to the analysis, SF-RTAs in China have special statistic characteristics. Data distribution in Fig. 2 and Fig. 3 shows the number SF-RTAs occurred in western region of China is the most, especially in southwest region. According to the statistics of China's Ministry of Public Security (Ministry of Public Security Traffic Management Bureau, 2015), the provinces in south-west region have more hilly land, where topography, meteorology, hydrology, and geological structure conditions are different with other places. These reasons may create dangerous driving conditions and result in more SF-RTAs. Thus, it is needed to deeply analyze accident mechanism for western provinces, and then put forward targeted strategies for preventing SF-RTAs.

The analysis for time distribution of SF-RTAs (as shown in Fig. 4) shows that SF-RTAs occurred between 6:00 to 8:00 and between 12:00 to $16: 00$ are more than in other time periods. This is because 6:00 to 8:00 belongs to morning rush hour of China, and traffic volume usually increases in this period. During 12:00 and 16:00, drivers are more likely to feel drowsy, and maybe cannot focus on driving. These may make SF-RTAs occurred in these 
periods more than in other time periods. Fig. 5 indicates that SF-RTAs occurred on Saturday are more than on other weekdays. As we all known, Saturday is the first day of weekend in China, thus, more people choose this day to go out for travel. On this day, the traffic volume of the roads increases and traffic problems, such as traffic congestion, traffic delay and road rage of drivers are more serious. These can result in the number of SF-RTAs occurred on Saturday increases. In addition, Fig.6 demonstrates SF-RTAs occurred in August and February is more than in other months. This is because, in China, August is the hottest month, when drivers are easily distracted in driving. And the spring festival of China (i.e. the Chinese New Year) is usually in February, which is a traditional day for family reunions. Many people choose to go home or go out to travel. These may make the number of SF-RTAs occurred in above two months more than in other months. Based on the analysis above, we may be able to reduce SF-RTAs by strengthening traffic control measures at accident prone periods and increasing traffic controllers in key and dangerous road sections.

Fig. 7 shows accidents related to run-off-road are the most. Thus, we'd better deeply analyze accident causes of run-off-road accidents, and take appropriate methods to contain devastating accidents with group deaths and accidents. The analysis of Fig. 8 shows that large buses and medium buses more easily result in SF-RTAs. That is, if we want to reduce SF-RTAs, it is important to enhance supervision of large buses and medium buses and strengthen the safety education and training to these vehicle drivers. Besides, Fig. 9 indicates SF-RTAs are more likely to happen on national roads and province roads. It suggests that, to control and decrease SF-RTAs, an effective way is to increase safety control equipments and put forward effective strategies for national and province roads.

The main factors influencing SF-RTAs are analyzed by linear regression and decision tree methods in this study. Table 3 and Fig. 10 indicate that the total population (H1) and substandard highway mileage (R4) have the strongest influence on the number of SF-RTAs in each province. The positive coefficients of H1 and R4 imply that the number of SF-RTAs increases with increase in H1 and R4. That is, if we want to reduce the number of SFRTAs, another method is to strengthen the safety education to the drivers in the provinces with larger population and decrease the mileage of substandard highway in these areas.

We hope this analysis can attract attention of traffic administrative department and be helpful to reduce SF-
RTAs, ensure traffic safety, and protect human lives and properties in China. In addition, one of the main limitations of this study is the unavailability of a long enough time series in the period analyzed. More regional data (such as weather information, motorization, use of seat belts, quality of the road network, etc.) will also help improve the analysis of serious fatal road traffic accidents (SF-RTAs) in China. Furthermore, we will choose more variables and analyze the relationship between the SFRTAs and various variables, and then investigate the mechanism of SF-RTAs for each province and propose more effective measures to prevent SF-RTAs.

\section{Acknowledgment}

This work was supported by the Opening Project of Key Laboratory of Road Safety Technologies, Ministry of Transport, P.R.China (2015RST06) and the Fundamental Research Funds for the National-level Research Institutes (Z2060302150009038).

\section{References}

1. WHO. Global status report on road safety, (2015).

2. M. Stanislav, S. Henrikas, Transport 28, 108-115, (2013).

3. The Ministry of Public Security Traffic Management Bureau. Road accident statistics annual report of the people's republic of China (2004-2014).

4. D. E. Clark, B. M. Cushing, Accident Analysis Prevention 36, 967-972, (2004).

5. R. Fu, Y. Guo, W. Yuan, H. Feng, Y. Ma, Safety Science 49, 416-420, (2011).

6. M. G. Washington, M. G. Karlaftis, F. L, Mannering, statistical and econometric methods for transportation data analysis (Chapman \& Hall, London, 2003).

7. P. Kitsantas, M. Hollander, L. Li, Artificial Intelligence in Medicine 38, 275-289, (2006).

8. T. S. Lee, C. C. Chiu, Y. C. Chou, C. J. Lu, Computational Statistics and Data Analysis 50, 11131130, (2006).

9. L. Breiman, J. Friedman, C. J. Stone and R. A. Olshen, Classification and regression trees (Chapman \& Hall, London, 1984).

10. L. Y. Chang, J. T. Chen, Safety Science 51, 17-22, (2012) .

11. O. de Juan, O. de Rocio and F. J. Calvo, Expert Systems with Applications 39, 11164-11171, (2012). 